\title{
Nonlinear Optimization for Polygonalization
}

\author{
Truong Kieu Linh ${ }^{1}$ and Atsushi Imiya ${ }^{2,3}$ \\ 1 School of Science and Technology, Chiba University, Japan \\ ${ }^{2}$ National Institute of Informatics, Japan \\ 3 Insutitute of Media and Information Technology \\ Chiba University, Japan
}

\begin{abstract}
In this paper, we first derive a set of inequalities for the parameters of a Euclidean line from sample pixels, and an optimization criterion with respect to this set of constraints for the recognition of the Euclidean line. Second, using this optimization problem, we prove uniqueness and ambiguity theorems for the reconstruction of a Euclidean line. Finally, we develop a polygonalization algorithm for the boundary of a discrete shape.
\end{abstract}

\section{Introduction}

In this paper, we aim to develop a polygonalization algorithm for the boundary of a discrete shape. For the reconstruction of a smooth boundary from sample points, the polygonalization on a plane is the first step. Following the polygonalization, we estimate the geometric features of a figure, such as the normal vector at each point on the boundary, and the length and area of planar shapes. We first derive a set of inequalities for the parameters of a Euclidean line from sample points, and an optimization criterion with respect to this system of inequalites. Second, we develop an algorithm for the computation of parameters of the Euclidean lines from pixels on a plane. Finally using this algorithm, we introduce a polygonalization algorithm. There are basically three types of models for the expression of a linear manifold in the grid space, supercover, standard, and naive models [1]. We deal with the supercover model on a plane.

Recently, a linear-programming based method for the recognition of linear manifolds has been proposed [23]. This method is based on the mathematical property that a point set determines a system of linear inequalities for the parameters of a linear manifold, and the recognition process for a linear manifold is converted to the computation of the feasible region for this system of inequalities. The other class for recognition of a linear manifold is based on the binary relation among local configurations of pixels in $3 \times 3$, which characterize the local properties of the discrete linear manifold 445|6|7. Our method proposed in this paper is based on the former method for the derivation of constraints on parameters of the Euclidean line that passes through pixels. Furthermore, we derive a minimization criterion for the parameters of the Euclidean line with respect to the constraints yielded from sets of pixels on a plane. 


\section{Optimization Problem for Recognition}

Setting $\boldsymbol{p}=\left(p_{1}, p_{2}\right)^{\top}$ to be a point in $\mathbf{Z}^{2}$, a hypercube,

$$
\mathbf{v}(\boldsymbol{p})=\left\{\boldsymbol{x}|| x_{i}-p_{i} \mid \leq \frac{1}{2}\right\}
$$

for $\boldsymbol{x}=\left(x_{1}, x_{2}\right)^{\top}$ is called a pixel of $\mathbf{Z}^{2}$. Hereafter, $P=\left\{\boldsymbol{x}_{i}\right\}_{i=1}^{k}$ stands for the set of the centers of the pixels $V=\left\{\mathbf{v}\left(\boldsymbol{x}_{i}\right)\right\}_{i=1}^{N}$. For $\boldsymbol{a}=(a, b)^{\top} \in \mathbf{Z}^{2}$ and $\mu \in \mathbf{Z}$, the supercover of the line $\boldsymbol{a}^{\top} \boldsymbol{x}+\mu=0$ is the collection of pixels which satisfy the inequality

$$
\left|\boldsymbol{a}^{\top} \boldsymbol{x}+\mu\right| \leq \frac{1}{2}|\boldsymbol{a}|_{1}
$$

where $|\boldsymbol{x}|_{1}$ is the $l_{1}$ norm of vector $\boldsymbol{x}$. Setting $\boldsymbol{s}_{\boldsymbol{x}}=\left(\operatorname{sgn} x_{1}, \operatorname{sgn} x_{2}\right)^{\top}$ the $l_{1}$-norm is expressed as $|\boldsymbol{x}|_{1}=\boldsymbol{s}_{\boldsymbol{x}}^{\top} \boldsymbol{x}$, since $|\boldsymbol{x}|_{1}=\sum_{i=1}^{n}\left|x_{i}\right|$. This expression derives the expression.

$$
-\frac{1}{2} \boldsymbol{a}^{\top} \boldsymbol{s} \boldsymbol{a} \leq \boldsymbol{a}^{\top} \boldsymbol{x}+\mu \leq \frac{1}{2} \boldsymbol{a}^{\top} \boldsymbol{s} \boldsymbol{a} .
$$

Recognition of a Euclidean line is stated as the following problem.

Problem 1 For a collection of sample points $\boldsymbol{x}_{i}$ for $i=1,2, \cdots, N$, if there exists a Euclidean line whose supercover contains all pixels $\left\{\mathbf{v}\left(\boldsymbol{x}_{i}\right)\right\}_{i=1}^{N}$, compute parameters $\boldsymbol{a}$ and $\mu$.

This problem is mathematically equivalent to finding parameters $\boldsymbol{a}$ and $\mu$ which satisfy the system of inequalities

$$
\left|\boldsymbol{a}^{\top} \boldsymbol{x}_{i}\right| \leq \frac{1}{2}|\boldsymbol{a}|_{1}, i=1,2, \cdots, N
$$

Equation (4) is equivalent to the system of inequalities

$$
\boldsymbol{a}^{\top} \boldsymbol{x}_{i}+\mu \geq-\frac{1}{2}|\boldsymbol{a}|_{1}, \quad-\boldsymbol{a}^{\top} \boldsymbol{x}_{j}-\mu \geq-\frac{1}{2}|\boldsymbol{a}|_{1}, \quad i, j=1,2, \cdots, N .
$$

Furthermore, eq. (5) derives the system of inequalities

$$
\boldsymbol{a}^{\top}\left(\boldsymbol{x}_{i j}-\boldsymbol{s}_{\boldsymbol{a}}\right) \geq 0,-\boldsymbol{a}^{\top} \boldsymbol{x}_{i}-\frac{1}{2}|\boldsymbol{a}|_{1} \leq \mu \leq-\boldsymbol{a}^{\top} \boldsymbol{x}_{i}+\frac{1}{2}|\boldsymbol{a}|_{1},
$$

for $i, j=1,2, \cdots, k, i \neq j$, where $\boldsymbol{x}_{i j}=\boldsymbol{x}_{i}-\boldsymbol{x}_{j}$. Since the vector $\boldsymbol{x}_{i j}+\boldsymbol{s}_{\boldsymbol{a}}$, such that $i \neq j$, is a constant for $i, j=1,2, \cdots, N$, eq. (6) determines a cone in $\mathbf{Z}^{2}$, that is, a integer cone in $\mathbf{R}^{2}$, depending on each $\frac{1}{2}$-space in which $\boldsymbol{a}$ lies. Since

$$
\frac{1}{2}|\boldsymbol{a}|_{1} \leq \frac{1}{2}\left(|\boldsymbol{a}|_{1}+|\mu|\right) \leq|\boldsymbol{a}|_{1}+|\mu|,
$$

we solve the following problem. 
Problem 2 Find $\boldsymbol{a} \in \mathbf{Z}^{2}$ and $\mu \in \mathbf{Z}$ which minimizes $z=y+|\mu|$, for $z \in \mathbf{Z}$ with respect to

$$
-\boldsymbol{a}^{\top} \boldsymbol{x}_{i}+\frac{1}{2} y \leq \mu \leq \boldsymbol{a}^{\top} \boldsymbol{x}_{i}+\frac{1}{2} y,
$$

and for $y$ which minimizes $y=|\boldsymbol{a}|_{1}$, for $\boldsymbol{a} \in \mathbf{Z}^{2}$ with respect to

$$
\boldsymbol{a}^{\top} \boldsymbol{y}_{i j} \geq 0, i, j=1,2, \cdots, N, i \neq j,
$$

where $\boldsymbol{y}_{i j}=\boldsymbol{x}_{i}-\boldsymbol{x}_{j}+\boldsymbol{s}_{\boldsymbol{a}}$.

For the supercover of a line, we have the following theorem.

Theorem 1 Pixels $V\left(\boldsymbol{x}_{i}\right)$ for $\left|\boldsymbol{a}^{\top} \boldsymbol{x}_{i}+\mu\right| \leq \frac{1}{2}|\boldsymbol{a}|_{1}$, if $|\boldsymbol{a}|_{1}=2 n$, then only $\boldsymbol{a}^{\top} \boldsymbol{x}+$ $\mu=0$ passes through all $V\left(\boldsymbol{v}_{i}\right)$, and if $|\boldsymbol{a}|_{1}=2 n+1$, for $|\gamma| \leq \frac{1}{2}$, all $\boldsymbol{a}^{\top} \boldsymbol{x}+\mu+\gamma=$ 0 passes through all pixels $V\left(\boldsymbol{x}_{i}\right)$ for $\left|\boldsymbol{a}^{\top} \boldsymbol{x}_{i}+\mu\right| \leq \frac{1}{2}|\boldsymbol{a}|_{1}$.

(Proof) If $|\boldsymbol{a}|=2 n$, then we have $0 \leq \boldsymbol{a}^{\top}\left(\boldsymbol{x}_{i}-\frac{1}{2} \boldsymbol{e}\right)+\mu \leq n$. For $\boldsymbol{y}_{i}=\boldsymbol{x}_{i}-\frac{1}{2} \boldsymbol{e}$,

$$
\left\{\left.v\left(\boldsymbol{x}_{i}\right)\left|0 \leq \boldsymbol{a}^{\top} \boldsymbol{x}+\mu+\frac{1}{2}\right| \boldsymbol{a}\right|_{1} \leq|\boldsymbol{a}|_{1}\right\}=\left\{v(\boldsymbol{x})\left|0 \leq \boldsymbol{a} \top\left(\boldsymbol{x}_{i}+\frac{1}{2} \boldsymbol{e}\right)+\mu \leq\right| \boldsymbol{a}_{1} \mid\right\} .
$$

Furthermore, $\boldsymbol{a}^{\top}\left(\boldsymbol{x}-\frac{1}{2} \boldsymbol{e}\right)+\mu=0$ and $\boldsymbol{a}^{\top} \boldsymbol{x}+\mu=0$ are the same line. This geometric property leads to the first part of the theorem. If $|\boldsymbol{a}|_{1}=2 n+1$, we have $0 \leq \boldsymbol{a}^{\top} \boldsymbol{x}_{i}+\mu+\gamma+\frac{|\boldsymbol{a}|_{1}}{2} \leq|\boldsymbol{a}|_{1}$ for $|\gamma| \leq \frac{1}{2}$. Therefore, $\boldsymbol{a}^{\top} \boldsymbol{x}_{i}+\mu+\gamma=0$ and $\boldsymbol{a}^{\top} \boldsymbol{x}_{i}+\mu=0$ determine the same set of pixels. This geometric property leads to the second part of the theorem.

(Q.E.D.)

For $|\boldsymbol{a}|_{1}=2 n+1$, lines exist in a strip whose center is $\boldsymbol{a}^{\top} \boldsymbol{x}+\mu=0$ and width is $\frac{1}{2\left|\boldsymbol{a}^{\top} \boldsymbol{a}\right|}$. For an integer $\lambda$ if $\lambda \mu+\lambda \gamma$ is an integer, both $\boldsymbol{a}^{\top} \boldsymbol{x}+\mu+\gamma=0$ and $\lambda \boldsymbol{a}^{\top} \boldsymbol{x}+\lambda \mu+\lambda \gamma=0$ are the same line. Therefore, $|\lambda|>1$, since

$$
|\boldsymbol{a}|_{1}+|\mu|<|\lambda|\left(\boldsymbol{a}_{1}+|\mu|\right)<\left|\boldsymbol{a}^{\prime}\right|_{1}
$$

for $\boldsymbol{a}^{\prime}=\left(\lambda \boldsymbol{a}^{\top}, \lambda(\mu+\gamma)\right)^{\top}$. This inequality geometrically means that the line which minimizes $\left(|\boldsymbol{a}|_{1}+|\mu|\right)$ is the central lines in the strip region. This geometric property shows the validity of our minimization algorithm. Therefore, we have the following theorem.

Theorem 2 If and only if $|\boldsymbol{a}|_{1}=2 n$, the supercover $\left|\boldsymbol{a}^{\top} \boldsymbol{x}+\mu\right| \leq \frac{1}{2}|\boldsymbol{a}|_{1}$ contains $2 \times 2$ squares.

(Proof) If $|\boldsymbol{a}|_{1}=2 n$, the Euclidean line $\boldsymbol{a}^{\top} \boldsymbol{x}+\mu=0$ passes through the point $\boldsymbol{x}+\frac{1}{2} \boldsymbol{e}$ for $\boldsymbol{x} \in \boldsymbol{Z}^{n}$. Moreover, for pixels $v(\boldsymbol{x})$ and $v\left(\boldsymbol{x}+\boldsymbol{e}_{i}\right)$, if $\left|\boldsymbol{a}^{\top} \boldsymbol{x}+\mu\right| \leq \frac{1}{2}|\boldsymbol{a}|_{1}$, the Euclidean line $\boldsymbol{a}^{\top} \boldsymbol{x}+\mu=0$ passes through the point $\boldsymbol{x}+\frac{1}{2} \boldsymbol{e}$, and $|\boldsymbol{a}|_{1}=2 n$. (Q.E.D.)

This theorem leads to the conclusion that, for a supercover, $2 \times 2$ squares guarantee the uniqueness of the Euclidean reconstruction of a plane.

For $\operatorname{gcd}(a, b, \mu)=1$, let $\operatorname{gcd}(a, b)=g, a^{\prime}=\frac{a}{g}$, and $b^{\prime}=\frac{b}{g}$. For the supercover of the line $L, a x+b y+\mu=0$, elementary number theory derives relations in tables 1 and 2, on the uniquness of the Euclidean reconstruction of lines with 
Table 1. Reconstruction of a line from the supercover with bubbles.

\begin{tabular}{|c|c|c|c|}
\hline $\operatorname{gcd}(a, b)$ & $a+b, a^{\prime}+b^{\prime}$ & Equivalent line & Universal line \\
\hline$g=1$ & $a+b:$ even & $L$ & $\emptyset$ \\
\hline$g=2$ & $a^{\prime}+b^{\prime}:$ odd & $L$ & $\emptyset$ \\
\hline$g>2$ & $\times$ & $\times$ & $\times$ \\
\hline
\end{tabular}

Table 2. Reconstruction of a line from the supercover without bubbles.

\begin{tabular}{|c|c|c|}
\hline Center line & Equivalent line & Universal line \\
\hline \multicolumn{3}{|c|}{$\operatorname{gcd}(a, b)=1 a+b=$ odd } \\
\hline$L$ & $\begin{array}{c}k a x+k b y+k \mu+k \varepsilon=0 \\
\text { where } \\
k \in Z,|\varepsilon|<\frac{1}{2}, k \varepsilon \in Z\end{array}$ & $2 a x+2 b y+2 \mu \pm 1$ \\
\hline \multicolumn{3}{|c|}{$\operatorname{gcd}(a, b)=2 a^{\prime}+b^{\prime}=$ odd } \\
\hline$L$ & $\begin{array}{c}k a x+k b y+k \mu+k \varepsilon=0 \\
\text { where } \\
k \in Z,|\varepsilon|<1, \quad k \varepsilon \in Z\end{array}$ & $a x+b y+\mu \pm 1=0$ \\
\hline \multicolumn{3}{|c|}{$\operatorname{gcd}(a, b)>2 a^{\prime}+b^{\prime}=$ odd } \\
\hline $\begin{array}{c}a^{\prime} x+b^{\prime} y+\mu^{\prime}=0 \\
\text { where } \mu^{\prime} \in Z \\
\frac{\mu}{g}-\frac{1}{2}<\mu^{\prime}<\frac{\mu}{g}+\frac{1}{2}\end{array}$ & $\begin{array}{c}k a^{\prime} x+k b^{\prime} y+k \mu^{\prime}+k \varepsilon=0 \\
\text { where } \\
k \in Z,|\varepsilon|<\frac{1}{2}, \quad k \varepsilon \in Z\end{array}$ & $2 a^{\prime} x+2 b^{\prime} y+2 \mu^{\prime} \pm 1$ \\
\hline \multicolumn{3}{|c|}{$\operatorname{gcd}(a, b)>2, a^{\prime}+b^{\prime}=$ even } \\
\hline $\begin{array}{c}2 a^{\prime} x+2 b^{\prime} y+2 \mu^{\prime}+1= \\
\text { where } \mu^{\prime} \in Z \\
\frac{\mu}{g}-1<\mu^{\prime}<\frac{\mu}{g}\end{array}$ & $\begin{array}{c}k a^{\prime} x+k b^{\prime} y+k \mu^{\prime}+k \varepsilon=0 \\
\text { where } \\
k \in Z, 0<\varepsilon<1, \quad k \varepsilon \in Z\end{array}$ & $\begin{array}{c}a^{\prime} x+b^{\prime} y+\mu^{\prime}=0 \\
\text { and } \\
a^{\prime} x+b^{\prime} y+\mu^{\prime}+1=0\end{array}$ \\
\hline
\end{tabular}

bubbles and with out bubples, respectively, from the geometrical and algebraic properties of bubbles. In the tables $Q$ is the set of all quotient numbers

In tables, the center line of the supercover $L$ is the line which minimizes the optimization criterion. The equivalent lines of $L$ are lines which define the same supercover with $L$. The universal lines of $L$ are the lines which contain all pixels of the supercover of $L$. The supercover of the universal line of $L$ always contains bubbles. These relations imply that if $\operatorname{gcd}(a, b)=1$, the line which minimizes the criterion is uniquely computed. Furthermore, if $a+b=$ odd, the line reconstructed from the supercover does not pass through the corners of pixels.

\section{Algorithm for Line Recognition}

Generally, we can set $a>0$ and $b>0$. Then, we have the following two equations,

$$
\text { case } 1: 0 \leq a x+b y+\mu+\frac{a+b}{2} \leq a+b
$$




$$
\text { case } 2: 0 \leq a x-b y+\mu+\frac{a+b}{2} \leq a+b \text {. }
$$

We set $X_{i j}=x_{i}-x_{j}+1, Y_{i j}=y_{i}-y_{j}+1$, for

$$
P=\left\{\boldsymbol{x}_{i}=\left(x_{i}, y_{i}\right)^{\top} \mid x_{i}, y_{i} \in Z, i=1,2, \cdots, N\right\},
$$

where $x_{1} \leq x_{2} \leq x_{3} \leq \cdots \leq x_{N}$. We define sets of points as

$$
\begin{aligned}
H & =\left\{\left(X_{i j}, Y_{i j}\right)^{\top} \mid i \neq j, i, j=1,2, \cdots, n\right\}, \\
Q_{++} & =\left\{\left(X_{i j}, Y_{i j}\right)^{\top} \mid\left(X_{i j}, Y_{i j}\right)^{\top} \in H, X_{i j}>0, Y_{i j}>0, i \neq j\right\}, \\
Q_{--} & =\left\{\left(X_{i j}, Y_{i j}\right)^{\top} \mid\left(X_{i j}, Y_{i j}\right)^{\top} \in H, X_{i j}<0, Y_{i j}<0, i \neq j\right\}, \\
Q_{+-} & =\left\{\left(X_{i j}, Y_{i j}\right)^{\top} \mid\left(X_{i j}, Y_{i j}\right)^{\top} \in H, X_{i j}>0, Y_{i j}<0, i \neq j\right\}, \\
Q_{-+} & =\left\{\left(X_{i j}, Y_{i j}\right)^{\top} \mid\left(X_{i j}, Y_{i j}\right)^{\top} \in H, X_{i j}<0, Y_{i j}>0, i \neq j\right\}, \\
Q_{0 X} & =\left\{\left(X_{i j}, Y_{i j}\right)^{\top} \mid\left(X_{i j}, Y_{i j}\right)^{\top} \in H, X_{i j}=0, i \neq j\right\}, \\
Q_{0 Y} & =\left\{\left(X_{i j}, Y_{i j}\right)^{\top} \mid\left(X_{i j}, Y_{i j}\right)^{\top} \in H, Y_{i j}=0, i \neq j\right\} .
\end{aligned}
$$

For these sets of points, if at least one of the four conditions

1. $Q_{--} \neq \emptyset$,

2. $\forall\left(X_{i j}, Y_{i j}\right)^{\top} \in Q_{0 X}, Y_{i j} \leq 0$,

3. $\forall\left(X_{i j}, Y_{i j}\right)^{\top} \in Q_{0 Y}, X_{i j} \leq 0$,

4. $Q_{+-} \neq \emptyset, Q_{-+} \neq \emptyset$, and

$$
\min \left(-\frac{X_{i j}}{Y_{i j}}\right)_{\mid\left(X_{i j}, Y_{i j}\right)^{\top} \in Q_{+-}}<\max \left(-\frac{X_{n m}}{Y_{n m}}\right)_{\mid\left(X_{n m}, Y_{n m}\right)^{\top} \in Q_{-+}}
$$

is satisfied, the system of inequalities has no solution. Therefore, we have the following theorem.

\section{Theorem 3 If}

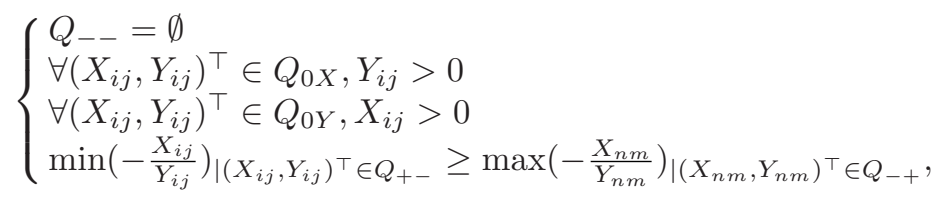

then the point set $P$ is the supercover of the Euclidean line $a x+b y+\mu=0$ for $a>0$ and $b>0$ which lie in the region

$$
\widehat{X_{i j}} a+\widehat{Y_{i j}} b \geq 0, \widehat{X_{m n}} a+\widehat{Y_{m n}} b \geq 0,
$$

where

$$
\begin{aligned}
\left(\widehat{X_{i j}}, \widehat{Y_{i j}}\right) & =\min \left(-\frac{X_{i j}}{Y_{i j}}\right)_{\mid\left(X_{i j}, Y_{i j}\right)^{\top} \in Q_{+-}} \\
\left(\widehat{X_{n m}}, \widehat{Y_{n m}}\right) & =\max \left(-\frac{X_{n m}}{Y_{n m}}\right)_{\mid\left(X_{n m}, Y_{n m}\right)^{\top} \in Q_{-+}}
\end{aligned}
$$


For $\boldsymbol{x}=\left(x_{i}, y_{i}\right)^{\top}$, we set $X_{i j}=\left(x_{i}-x_{j}+1\right.$ and $Y_{i j}=y_{j}-y_{i}+1$. Then, for

$$
\begin{aligned}
\bar{H} & =\left\{\left(X_{i j}, Y_{j i}\right)^{\top} \mid i \neq j, i, j=1,2, \cdots, N\right\} \\
\overline{Q_{++}} & =\left\{\left(X_{i j}, Y_{j i}\right)^{\top} \mid\left(X_{i j}, Y_{j i}\right)^{\top} \in H, X_{i j}>0, Y_{j i}>0, i \neq j\right\} \\
\overline{Q_{--}} & =\left\{\left(X_{i j}, Y_{j i}\right)^{\top} \mid\left(X_{i j}, Y_{j i}\right)^{\top} \in H, X_{i j}<0, Y_{j i}<0, i \neq j\right\} \\
\overline{Q_{+-}} & =\left\{\left(X_{i j}, Y_{j i}\right)^{\top} \mid\left(X_{i j}, Y_{j i}\right)^{\top} \in H, X_{i j}>0, Y_{j i}<0, i \neq j\right\} \\
\overline{Q_{-+}} & =\left\{\left(X_{i j}, Y_{j i}\right)^{\top} \mid\left(X_{i j}, Y_{j i}\right)^{\top} \in H, X_{i j}<0, Y_{j i}>0, i \neq j\right\} \\
\overline{Q_{0 X}} & =\left\{\left(X_{i j}, Y_{j i}\right)^{\top} \mid\left(X_{i j}, Y_{j i}\right)^{\top} \in H, X_{i j}=0, i \neq j\right\} \\
\overline{Q_{0 Y}} & =\left\{\left(X_{i j}, Y_{j i}\right)^{\top} \mid\left(X_{i j}, Y_{j i}\right)^{\top} \in H, Y_{j i}=0, i \neq j\right\},
\end{aligned}
$$

if at least one of the four conditions

1. $\overline{Q_{--}} \neq \emptyset$,

2. $\forall\left(X_{i j}, Y_{j i}\right)^{\top} \in Q_{0 X}, Y_{j i} \leq 0$,

3. $\forall\left(X_{i j}, Y_{j i}\right)^{\top} \in Q_{0 Y}, X_{i j} \leq 0$,

4. $\overline{Q_{+-}} \neq \emptyset, \overline{Q_{-+}} \neq \emptyset$, and

$$
\min \left(-\frac{X_{i j}}{Y_{j i}}\right)_{\mid\left(X_{i j}, Y_{j i}\right)^{\top} \in \overline{Q_{+-}}}<\max \left(-\frac{X_{m n}}{Y_{n m}}\right)_{\mid\left(X_{m n}, Y_{n m}\right)^{\top} \in \overline{Q_{-+}}} .
$$

is satisfied, the system of inequalities has no solution. Therefore, we have the following theorem.

\section{Theorem 4 If}

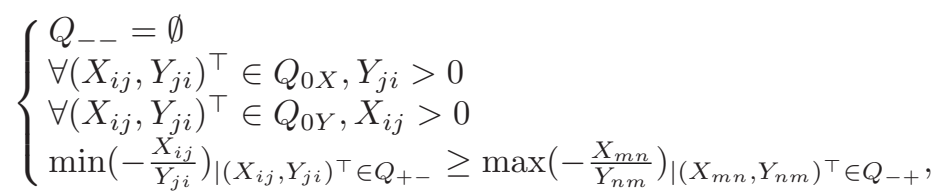

then the point set $P$ is the supercover of the Euclidean line ax $-b y+\mu=0$ for $a>0$ and $b>0$ which lie in the region

$$
\widehat{X_{i j}} a+\widehat{Y_{j i}} b \geq 0, \widehat{X_{m n}} a+\widehat{Y_{n m}} b \geq 0
$$

where

$$
\begin{aligned}
\left(\widehat{X_{i j}}, \widehat{Y_{j i}}\right) & =\min \left(-\frac{X_{i j}}{Y_{j i}}\right)_{\mid\left(X_{i j}, Y_{j i}\right)^{\top} \in Q_{+-}} \\
\left(\widehat{X_{m n}}, \widehat{Y_{n m}}\right) & =\max \left(-\frac{X_{m n}}{Y_{n m}}\right)_{\mid\left(X_{m n}, Y_{n m}\right)^{\top} \in Q_{-+}}
\end{aligned}
$$

Assuming that the feasible region of the inequalites is the cone bounded by two lines $\alpha_{1} x+\beta_{1} y \leq 0$ and $\alpha_{2} x+\beta_{2} y \geq 0$ Since $a>0$ and $b>0$, the minimum of $|a|+|b|$ is computed by the following algorithm. 
step 1: Set $a+b=k$

step 2: The line $a+b=k$ crosses at points $\left(a_{1}, b_{1}\right)^{\top}$ and $\left(a_{2}, b_{2}\right)^{\top}$ with $\alpha_{1} a+\beta_{1} b=0$ and $\alpha_{2} a+\beta_{2} b=0$, respectively. Then, we have $a_{1}=\frac{\beta_{1}}{\beta_{1}-\alpha_{1}} k$ and $a_{2}=\frac{\beta_{2}}{\beta_{2}-\alpha_{2}} k$.

step $3: k:=2$.

step 4: For $a=\left\{a \mid a_{1} \leq a \leq a_{2}, a \in Z\right\}, \min \{k\}, b=k-a$, if both $a$ and $b$ are integers then stop, else $k:=1$.

For $a x+b y+\mu=0, a>0$ and $b>0, \mu$ satisfies the inequality

$$
\max \left\{-\left(x_{i}+\frac{1}{2}\right) a-\left(y_{i}+\frac{1}{2}\right) b\right\} \leq \mu \leq \min \left\{\left(\frac{1}{2}-x_{i}\right) a+\left(\frac{1}{2}-y_{i}\right) b\right\} .
$$

Therefore,

- if $\max \left\{-\left(x_{i}+\frac{1}{2}\right) a-\left(y_{i}+\frac{1}{2}\right) b\right\} \geq 0, \mu=\max \left\{-\left(x_{i}+\frac{1}{2}\right) a-\left(y_{i}+\frac{1}{2}\right) b\right\}$.

- if $\min \left\{\left(\frac{1}{2}-x_{i}\right) a+\left(\frac{1}{2}-y_{i}\right) b\right\} \leq 0, \mu=\min \left\{\left(\frac{1}{2}-x_{i}\right) a+\left(\frac{1}{2}-y_{i}\right) b\right\}$.

- if $\max \left\{-\left(x_{i}+\frac{1}{2}\right) a-\left(y_{i}+\frac{1}{2}\right) b\right\}<0$ and $\min \left\{\left(\frac{1}{2}-x_{i}\right) a+\left(\frac{1}{2}-y_{i}\right) b\right\}>0$, $\mu=0$.

Furthermore, for $a x-b y+\mu=0$, where $a>0$ and $b>0, \mu$ satisfies the inequality

$$
\max \left\{-\left(x_{i}+\frac{1}{2}\right) a+\left(y_{i}-\frac{1}{2}\right) b\right\} \leq \mu \leq \min \left\{\left(\frac{1}{2}-x_{i}\right) a+\left(\frac{1}{2}+y_{i}\right) b\right\} .
$$

Therefore,

- if $\max \left\{-\left(x_{i}+\frac{1}{2}\right) a+\left(y_{i}-\frac{1}{2}\right) b\right\} \geq 0, \mu=\max \left\{-\left(x_{i}+\frac{1}{2}\right) a+\left(y_{i}-\frac{1}{2}\right) b\right\}$,

- if $\min \left\{\left(\frac{1}{2}-x_{i}\right) a+\left(\frac{1}{2}+y_{i}\right) b\right\} \leq 0, \mu=\min \left\{\left(\frac{1}{2}-x_{i}\right) a+\left(\frac{1}{2}+y_{i}\right) b\right\}$,

- if $\max \left\{-\left(x_{i}-\frac{1}{2}\right) a+\left(y_{i}+\frac{1}{2}\right) b\right\}<0$ and $\min \left(\frac{1}{2}-x_{i}\right) a+\left(\frac{1}{2}-y_{i}\right) b>0, \mu=0$.

\section{Polygonalization}

Using the optimization procedure for the recognition of a Euclidean line from a collection of pixels, in this section we develop an algorithm for the polygonalization of the discrete boundary of a binary shape. We assume that the 4-connected boundary is extracted by an appropriate boundary extraction method

Setting $\mathbf{P}$ to be a digital curve which is a sequence of 4-connected pixels, our problem is described as follows.

Problem 3 For a digital boundary curve $\mathbf{P}$, setting $\left\{\boldsymbol{p}_{i j}\right\}_{j=1}^{n(i)}=\mathbf{P}_{i}$, derive a partition of $\mathbf{P}, \mathbf{P}=\cup_{i=1}^{n} \mathbf{P}_{i}$, such that $\left|\mathbf{P}_{i} \cap \mathbf{P}_{i+1}\right|=\varepsilon$, where $\varepsilon$ is an appropriate integer, which minimizes

$$
z=\sum_{i=1}^{n}\left(\left|\boldsymbol{a}_{i}\right|_{1}+\mu_{i}\right)
$$

for the system of inequalities,

$$
\left|\boldsymbol{a}_{i}^{\top} \boldsymbol{x}_{i j}+\mu_{i}\right| \leq \frac{1}{2}\left|\boldsymbol{a}_{i}\right|_{1},
$$

for $i=1,2, \cdots, n$ and $j=1,2, \cdots, n(i)$. 
To solve this minimization problem, we prepare the following lemmas.

Lemma 1 Setting $\boldsymbol{p}_{1}=\left(x_{1}, y_{1}\right)^{\top}$ and $\boldsymbol{p}_{2}=\left(x_{2}, y_{2}\right)^{\top}$ to be a pair of points on supercover

$$
L(a, b, \mu)=\left\{x, y \in \mathbf{Z}|| a x+b y+\mu \mid \leq \frac{1}{2}(|a|+|b|)\right\}
$$

the number of pixels between $\boldsymbol{p}_{1}$ and $\boldsymbol{p}_{2}$ along this supercover is

$$
N\left(\boldsymbol{p}_{1}, \boldsymbol{p}_{2}\right)= \begin{cases}\left|\boldsymbol{p}_{1}-\boldsymbol{p}_{2}\right|+1 & \text { if }|a|+|b|=\text { odd } \\ \left|\boldsymbol{p}_{1}-\boldsymbol{p}_{2}\right|+\left[\frac{\left|x_{1}-x_{2}\right|}{|b|}\right]+\delta & \text { if }|a|+|b|=\text { even }\end{cases}
$$

where $\delta \in\{1,2\}$

Lemma 2 For a pair of points $\boldsymbol{p}_{1}=\left(x_{1}, y_{1}\right)^{\top}$ and $\boldsymbol{p}_{2}=\left(x_{2}, y_{2}\right)^{\top}$ on a supercover, setting $\boldsymbol{p}_{3}$ to be the center of a pixel on this supercover between these two points, we have the relation

$$
\left|\operatorname{det}\left(\boldsymbol{p}_{2}-\boldsymbol{p}_{3}, \boldsymbol{p}_{1}-\boldsymbol{p}_{3}\right)\right| \leq\left|\boldsymbol{p}_{2}-\boldsymbol{p}_{1}\right|_{1}
$$

Using these properties of the supercover, we introduce the following algorithm.

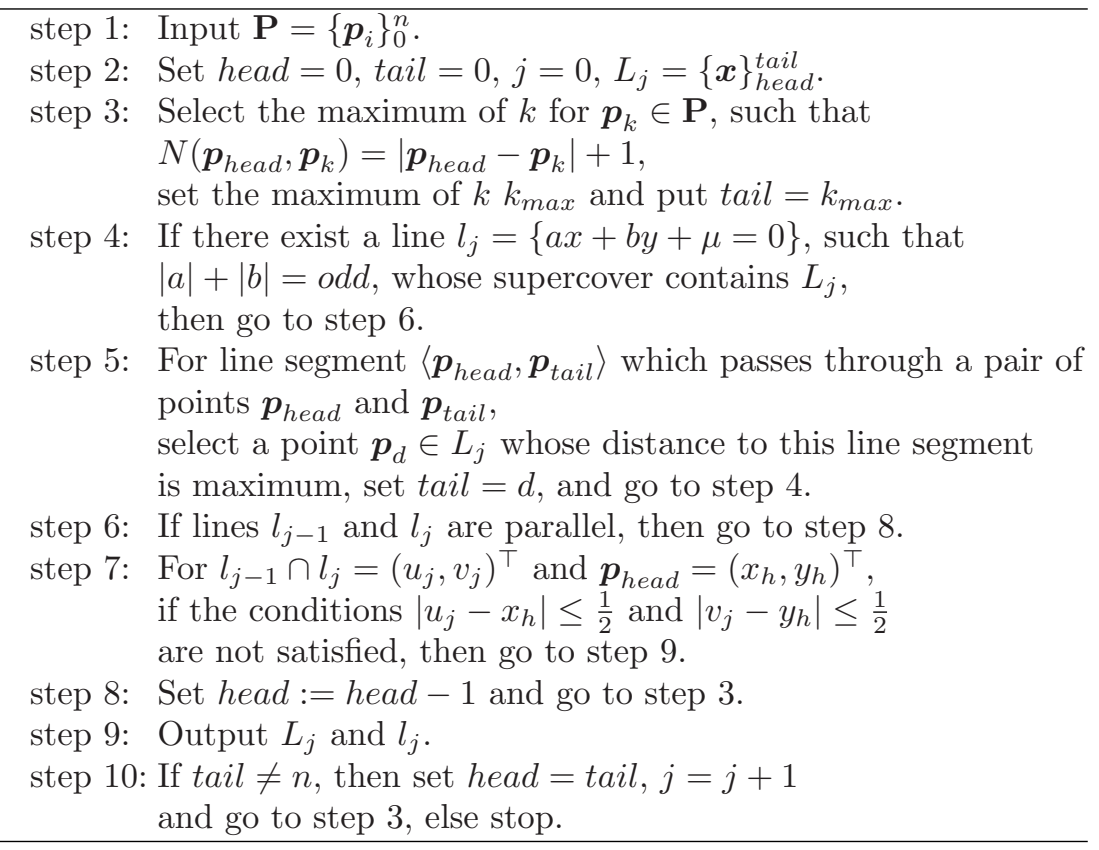

Geometrically, the algorithm detects the candidate of the minimum-lenght path between a pair of points on the digital boundary, examining the relation 


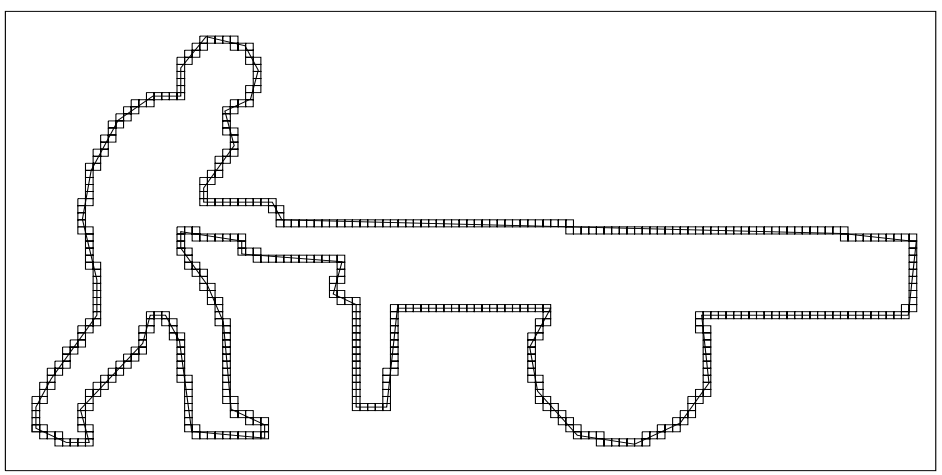

Fig. 1. Boundary pixels and the Euclidan polygon of SS253TL2 [8].

Table 3. Parameters of lines for the boundary of SS253TL2 8].

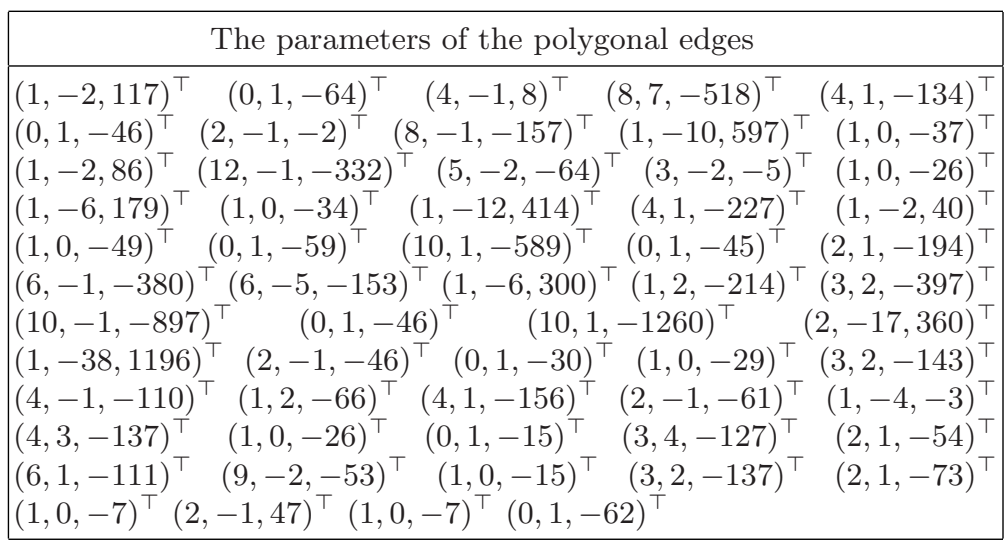

between numbers of pixels on a supercover. If this candidate determines a Euclidean line, then the algorithm computes the parameters solving the nonlinear optimization problem derived in the section 2 . If this candidate does not determine a Euclidean line, by separating the candidate path, the algorithm continues the same procedure in order to detect a Euclidean line. In this algorithm, steps 4, 7 , and 8 are achieved to fulfill the conditions that polygonal edges do not cross the corners of the pixels, that the successive two lines are not parallel, and that the polygonal vertices exist in the pixels of the digital boundary. According to the greedy property of the algorithm, this algorithm stops and fulfils the uniqueness of the solution for the starting point.

In Figure 1, we show a result of the polygonalization by this algorithm for a graphical ornament [8]. In Figure 1, the boundary is extracted from a binary graphical ornament and the polygonal curve is superimposed on the sequence of boundary pixels. Table 3 shows the parameters of the Euclidean lines for the polygonalization for the boundary. 


\section{Conclusions}

We developed an algorithm for the computation of the parameters of a Euclidean line from pixels on a plane. We also proved uniqueness and ambiguity theorems for the reconstruction of Euclidean lines. We have also developed an algorithm for the polygonalization of a digital 4-connected boundary of binary shapes.

The standard model of a discrete line is defined as

$$
0 \leq \boldsymbol{a}^{\top} \boldsymbol{x}+\mu<|\boldsymbol{a}|_{1}
$$

Assuming that $\boldsymbol{a}>0$, for a standard line, we have the system of inequalities,

$$
\boldsymbol{a}^{\top} \boldsymbol{x}_{i}+\mu \geq 0, \quad \boldsymbol{a}^{\top}\left(\boldsymbol{e}-\boldsymbol{x}_{j}\right)-\mu>0, \quad-\boldsymbol{a}^{\top} \boldsymbol{x}_{i} \leq \mu<\boldsymbol{a}^{\top}\left(\boldsymbol{e}-\boldsymbol{x}_{j}\right)
$$

from the collection of sample points $P$. Therefore, the feasible region of $\boldsymbol{a}$ is defined by the system of inequalities

$$
\boldsymbol{a}>0, \quad \boldsymbol{a}^{\top}\left(\boldsymbol{x}_{i}-\boldsymbol{x}_{j}+\boldsymbol{e}\right)>0 .
$$

Then, setting $\boldsymbol{x}_{i j}=\boldsymbol{x}_{i}-\boldsymbol{x}_{j}+\boldsymbol{e}$, the algorithm proposed in this paper recognizes a standard line and reconstructs a Euclidean line from sample pixels.

\section{References}

1. Andres, E., Nehlig, P., Francon, J., Supercover of straight lines, planes, and triangles, LNCS, 1347, 243-254, 1997.

2. Francon, J., Schramm, J.M., Tajine, M., Recognizing arithmetic straight lines and planes, LNCS, 1176, 141-150, 1996.

3. Buzer, L., An incremental linear time algorithm for digital line and plane recognition using a linear incrimental feasibility problem, LNCS, 2301, 372-381, 2002.

4. Barneva, R. P., Brimkov, V. E., Nehlig, P., Thin discrete triangular meshes, Theoretical Computer Science, 246, 73-105, 2000 D

5. Schramm, J.M., Coplanar tricubes, LNCS, 1347, 87-98, 1997.

6. Vittone, J., Chassery, J. M., Digital naive planes understanding, Proceedings of SPIE, 3811, 22-32, 1999.

7. Reveilles, J.-P., Combinatorial pieces in digital lines and planes, Proceedings of SPIE, 2573, 23-34, 1995.

8. SS253TL2 in Graphic Ornaments, The Pepin Press-Agile Rabbit Edition; Amsterdam, 2001. 